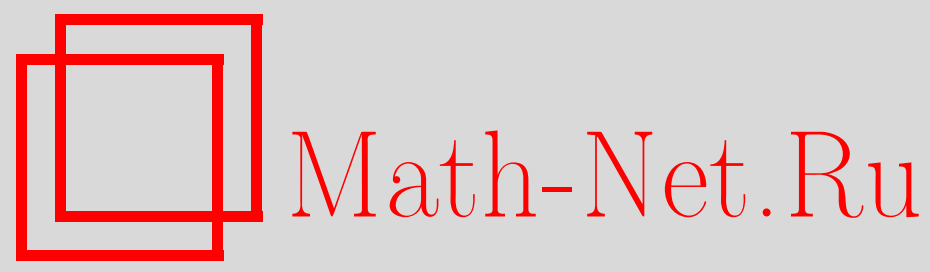

В. Н. Сорокин, Вполне интегрируемые нелинейные динамические системы типа цепочек Ленгмюра, Матем. заметки, 1997, том 62, выпуск 4, 588-602

DOI: https://doi.org/10.4213/mzm1641

Использование Общероссийского математического портала Math-Net.Ru подразумевает, что вы прочитали и согласны с пользовательским соглашением http://www . mathnet.ru/rus/agreement

Параметры загрузки:

IP : 52.6 .47 .48

26 апреля 2023 г., $14: 22: 31$

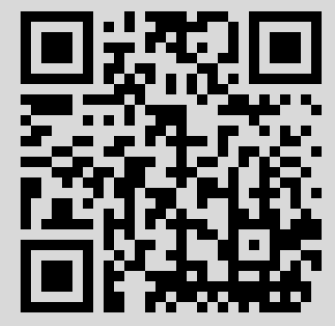




\section{ВПОЛНЕ ИНТЕГРИРУЕМЫЕ НЕЛИНЕЙНЫЕ ДИНАМИЧЕСКИЕ СИСТЕМЫ ТИПА ЦЕПОЧЕК ЛЕНГМЮРА}

\section{В. Н. Сорокин}

Решение задачи Коши для полубесконечной цепочки обыкновенных дифференциальных уравнений, впервые рассмотренной в 1987 году О. И. Богоявленским, получено в терминах разложения вектора марковских функций - резольвентных функций, - связанного с цепочкой несимметричного оператора, в многомерную непрерывную дробь по алгоритму Эйлера-Якоби-Перрона. Применяется метод обратной спектральной задачи, основанный на паре Лакса, теория совместных аппроксимаций Эрмита-Паде и метод Штурма-Лиувилля для разностных уравнений.

Библиографоия: 13 названий.

1. В этой работе мы проинтегрируем цепочку дифференциальных уравнений

$$
\dot{c}_{n}=c_{n}\left(c_{n+1} \cdots c_{n+r}-c_{n-1} \cdots c_{n-r}\right), \quad n \in \mathbb{Z}_{+}
$$

с граничньми условиями

$$
c_{-r}=0, \ldots, \quad c_{-1}=0
$$

и с начальными условиями $c_{n}(0)>0, n \in \mathbb{Z}_{+}$. Здесь $r-$ фиксированное натуральное число. Решение цепочки ищется при $t \geqslant 0$.

При $r=1$ система дифференциальных уравнений (1) - это хорошо известная цепочка Ленгмюра [1]. При $r>1$ цепочки (1) впервые ввел в рассмотрение и изучал О.И. Богоявленский в 1987 году [2], [3]. Была установлена связь этих цепочек с уравнением Кортевега-де Фриза, найдены соответствующие им пары Лакса, указан набор первых интегралов и предложена схема решения цепочек методом обратной спектральной задачи. Реализация этой схемы наталкивается на существенные трудности, связанные с изучением спектральных характеристик возникающих при этом несимметричных операторов. В последующих работах этот метод развивался в направлении, использующем матрицы Вейля. Укажем лишь две из последних таких работ [4], [5], где, в частности, интегрируются цепочки типа Тоды.

Мы проинтегрируем систему (1), (2), также используя метод обратной спектральной задачи для некоторого несимметричного разностного оператора, причем представим

Работа выполнена при поддержке Международного научного фонда и Правительства России, грант N7N300, фонда INTAS, грант № 93-219ext, фонда Deutcshe Forschungsgemeinschaft, грант AZ: 436 RUS 17/42/95, и Российского фонда фундаментальных исследований, грант № 96-01-00730. 
ответ в виде, вполне аналогичном классическому самосопряженному случаю. Необходимо отметить, что недавно А. И. Аптекаревым и В.А. Калягиным независимо также были получены новые результаты о цепочках, отличных от (1), но имеющих аналогичную структуру. Их методы исследования отличаются от наших (эти результаты еще не опубликованы).

Будем считать, что $r=2$. Переход к произвольному $r$ тривиален. Определим операцию деления вектора $g=\left(g^{(1)}, g^{(0)}\right)$ на вектор $d=\left(d^{(1)}, d^{(0)}\right)$ следующей формулой:

$$
\frac{g}{d}=\left(\frac{g^{(1)} d^{(0)}}{d^{(1)}}, \frac{g^{(0)}}{d^{(1)}}\right),
$$

если только $d^{(1)} \neq 0$. Обозначим через $\Delta$ множество в комплексной плоскости, состоящее из трех лучей, выходящих из нуля и проходящих через корни третьей степени из единицы.

Основным результатом работы является следующая

ТЕОремА. Задача Коши для бесконечной системы обыкновенных дифференииальных уравнений

$$
\dot{c}_{n}=c_{n}\left(c_{n+1} c_{n+2}-c_{n-1} c_{n-2}\right), \quad n \in \mathbb{Z}_{+},
$$

с граничными условиямм

$$
c_{-2}=0, \quad c_{-1}=0,
$$

начальные условия которой $c_{n}(0)$ образуют ограниченную последовательность положительных чисел, имеет единственное решение на промехутке $t \in[0,+\infty)$. Решением является ограниченная (при каждом $t$ ) последовательность положительных функиий $c_{n}(t)$, которая вычисляется по формуле

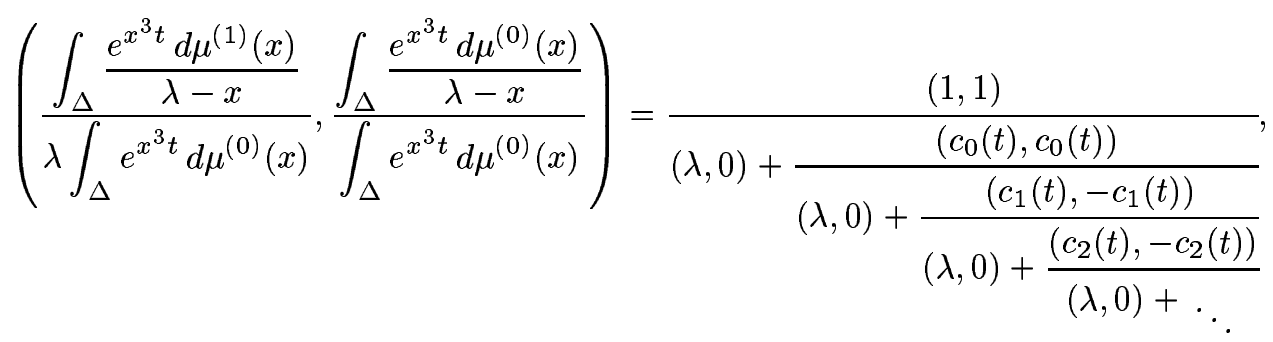

әде конечные полохительные борелевские меры $d \mu^{(0)}(x)$ и $d \mu^{(1)}(x)$ с общим компактным носителем на $\Delta$ единственным образом (с точностью до нормировки) определяются той же формулой (6) при $t=0$.

Таким образом, для того чтобы решить цепочку (4), (5), мы, используя известные начальные условия $c_{n}(0)$, суммируем непрерьвную дробь в (6) и, например по формуле Стилтьеса-Перрона, находим меры $d \mu^{(0)}, d \mu^{(1)}$. После чего, раскладывая левую часть (6) в непрерьвную дробь, получаем решение $c_{n}(t)$.

Доказательство теоремы завершается в п. 8 применением лемм, доказанных в п. 2-7. В п. 9 рассмотрен один конкретньй пример. 
2. Обозначим через $S$ множество всех последовательностей действительных чисел

$$
s=\left(s_{0}, s_{1}, s_{2}, \ldots\right)
$$

таких, что $s_{3 n+2}=0, n \in \mathbb{Z}_{+}$. Обозначим через $\stackrel{\circ}{S}$ множество всех последовательностей из $S$, нормированных условием $s_{0}=1$. Рассмотрим бесконечную матрицу

$$
\mathscr{H}^{(0)}=\left(\begin{array}{cccc}
s_{0} & s_{3} & s_{6} & \ldots \\
s_{1} & s_{4} & s_{7} & \ldots \\
s_{3} & s_{6} & s_{9} & \ldots \\
\ldots & \ldots & \ldots
\end{array}\right),
$$

а также матрицы $\mathscr{H}^{(1)}$ и $\mathscr{H}^{(2)}$, которые получаются из $\mathscr{H}^{(0)}$ вычеркиванием первой строки и первых двух строк соответственно.

Обобщенными определителями Ганкеля $\mathscr{H}_{n}^{(j)}$ будем назьвать главные миноры порядка $n$ матриц $\mathscr{H}^{(j)}, n=1,2,3, \ldots$, и по определению $\mathscr{H}_{0}^{(j)}=1$, где $j=0,1,2$.

Последовательность $s \in S$ назовем несингулярной, если все ее обобщенные определители отличны от 0 . Последовательность $s \in S$ назовем позитивной, если все ее обобщенные определители Ганкеля положительны:

$$
\mathscr{H}_{n}^{(j)}>0, \quad n \in \mathbb{Z}_{+}, \quad j=0,1,2 .
$$

Множество всех позитивных последовательностей обозначим через $S_{+}$. Множество всех позитивных последовательностей из $\stackrel{\circ}{S}$ обозначим через $\stackrel{\circ}{S}+$

Пусть $\mathbb{R}[\lambda]$ - линейное пространство всех многочленов с вещественными коэффициентами. Каждая последовательность $s \in S$ порождает линейньй функционал $\mathfrak{S}: \mathbb{R}[\lambda] \rightarrow \mathbb{R}$, ставящий в соответствие многочлену

$$
P(\lambda)=p_{n} \lambda^{n}+p_{n-1} \lambda^{n-1}+\cdots+p_{0}
$$

число

$$
\mathfrak{S}\{P(\lambda)\}=s_{n} p_{n}+s_{n-1} p_{n-1}+\cdots+s_{0} p_{0} .
$$

Функционал $\mathfrak{S}$ назовем позитивным (несингулярным $)$, если позитивна (несингулярна) порождающая этот функционал последовательность.

Будем обозначать через $\varepsilon=\exp \{2 \pi i / 3\}$ кубический корень из единицы и через $\mathbb{Z}_{3}-$ циклическую группу 3 -го порядка, состоящую из следующих преобразований комплексной плоскости: $z \mapsto \varepsilon^{l} z, l=0,1,2$.

Зафиксируем некоторый несингулярный функционал $\mathfrak{S}$ и поставим следующую задачу.

ЗАДА чА (Н). Для каждого $n \in \mathbb{Z}_{+}$требуется найти многочлен $H_{n}(\lambda)$ такой, что

1) $\operatorname{deg} H_{n}(\lambda) \leqslant n$;

2) $H_{n}(\lambda) \not \equiv 0$;

3) $H_{n}(\lambda)$ инвариантен относительно группы $\mathbb{Z}_{3}$, т.е. $H_{n}(\varepsilon \lambda)=\varepsilon^{n} H_{n}(\lambda)$;

4) $H_{n}(\lambda)$ удовлетворяет соотношениям ортогональности $\mathfrak{S}\left\{H_{n}(\lambda) \lambda^{k}\right\}=0$, когда $k=0, \ldots,[n / 2]-1$ и $n+k+1 \not \equiv 0(\bmod 3)$. 
ЛЕмма 1. Если $\mathfrak{S}$ - несингулярный функционал, то для каждого $n \in \mathbb{Z}_{+}$существует и притом только один (с точностью до нормировки) многочлен $H_{n}(\lambda)$ степени в точности п, удовлетворяющий условиям задачи (H).

ДокАЗАТЕЛЬСТво леммы следует из того, что условия задачи $(\mathrm{H})$ сводятся к системе линейных однородных уравнений относительно неизвестных коэффициентов многочлена $H_{n}(\lambda)$. При этом, приравнивая к 0 старшие коэффициенты многочлена $H_{n}(\lambda)$, мы будем получать системы, определителями которых служат обобщенные определители Ганкеля.

ЗАмЕчАнИЕ. Из доказательства леммы 1 вытекают детерминантные формулы для многочленов $H_{n}(\lambda)$. Например,

$$
H_{6 n}(\lambda)=\frac{h_{6 n}}{\mathscr{H}_{2 n}^{(0)}}\left|\begin{array}{cccc}
s_{0} & s_{3} & \ldots & s_{6 n} \\
s_{1} & s_{4} & \ldots & s_{6 n+1} \\
\ldots \ldots \ldots \ldots \ldots \ldots & \ldots \ldots \ldots \\
s_{3 n-2} & s_{3 n+1} & \ldots & s_{9 n-2} \\
1 & \lambda^{3} & \ldots & \lambda^{6 n}
\end{array}\right|
$$

где $h_{6 n}$ - старший коэффициент многочлена $H_{6 n}(\lambda)$. Аналогичные формулы имеют место и для других индексов.

Назовем многочлены $H_{n}(\lambda)$ инвариантными (относительно группы $\mathbb{Z}_{3}$ ) многочленами совместной ортогональности. Они служат естественным обобщением многочленов, ортогональных на всей вещественной оси с симметричной мерой.

До сих пор мы никак не фиксировали нормировку многочленов $H_{n}(\lambda)$. Будем обозначать через $\stackrel{\circ}{H}_{n}(\lambda)$ многочлены с единичным старшим коэффициентом. Существенную роль в дальнейшем будет играть некоторая специальная нормировка. Введем нормировочные постоянные

$$
m_{n}=\mathfrak{S}\left\{\stackrel{\circ}{H}_{n}(\lambda) \lambda^{[n / 2]}\right\}, \quad n \in \mathbb{Z}_{+},
$$

где [·] - целая часть числа. Из замечания к лемме 1 следует, что

$$
m_{3 n+j}=\frac{\mathscr{H}_{n+1}^{(j)}}{\mathscr{H}_{n}^{(j)}}, \quad j=0,1,2, \quad n \in \mathbb{Z}_{+} .
$$

Таким образом, функционал $\mathfrak{S}$ позитивный тогда и только тогда, когда все $m_{n}>0$, $n \in \mathbb{Z}_{+}$.

Пусть $\mathfrak{S}$ - позитивньй функционал. Многочлены $H_{n}(\lambda)$ будем назьвать нормированными, если их старшие коэффициенты вычисляются по формулам $h_{n}=m_{n}^{-2 / 3}$, $n \in \mathbb{Z}_{+}$.

Лемма 2. Если последовательность $s \in \stackrel{\circ}{S}_{+}$, то соответствующая ей последовательность нормированных инвариантных многочленов совместной ортогональности $\left\{H_{n}(\lambda)\right\}_{n=0}^{\infty}$ является единственным решением рекуррентного соотношения

$$
a_{n} H_{n+1}(\lambda)+b_{n-2} H_{n-2}(\lambda)=\lambda H_{n}(\lambda), \quad n \in \mathbb{Z}_{+},
$$

с начальными условиями

$$
H_{-2}(\lambda)=0, \quad H_{-1}(\lambda)=0, \quad H_{0}(\lambda)=1 .
$$


Коэффициенты рекуррентного соотношения определяются по формулам

$$
a_{n}=\frac{h_{n}}{h_{n+1}}, \quad b_{n}=\sqrt{a_{n} a_{n+1}}, \quad n \in \mathbb{Z}_{+} .
$$

ДокАЗАТЕЛЬСтво. Рассмотрим многочлен вида

$$
H(\lambda)=\lambda H_{n}(\lambda)-b H_{n-2}(\lambda)
$$

При любом выборе параметра $b$ этот многочлен удовлетворяет условиям 1)-3) задачи $(\mathrm{H})$ для индекса $n+1$, а также всем соотношениям ортогональности условия 4) за исключением, быть может, последнего:

$$
\mathfrak{S}\left\{H(\lambda) \lambda^{[n / 2]-1}\right\}=0
$$

Параметр $b$ найдем из условия (9), равносильного уравнению

$$
b \mathfrak{S}\left\{H_{n-2}(\lambda) \lambda^{[n / 2]-1}\right\}=\mathfrak{S}\left\{H_{n}(\lambda) \lambda^{[n / 2]}\right\} .
$$

Отсюда находим

$$
b=\frac{h_{n} m_{n}}{h_{n-2} m_{n-2}}=\left(\frac{h_{n-2}}{h_{n}}\right)^{1 / 2}
$$

Тогда $H(\lambda)=a H_{n+1}(\lambda)$, где постоянную $a$ находим из сравнения старших коэффициентов, а именно: $a=h_{n} / h_{n+1}$.

Построим из коэффициентов рекуррентного соотношения (7) бесконечную матрицу

$$
A=\left(\begin{array}{cccccc}
0 & a_{0} & & & & \\
0 & 0 & a_{1} & & 0 & \\
b_{0} & 0 & 0 & a_{2} & & \\
& b_{1} & 0 & 0 & a_{3} & \\
0 & & \ddots & \ddots & \ddots & \ddots
\end{array}\right)
$$

Если $H=\left(H_{0}, H_{1}, \ldots\right)^{T}$ - бесконечньй столбец многочленов $H_{n}(\lambda)$, то соотношение $(7)$ запишется в матричном виде $A H=\lambda H$.

Обобщенной матрицей Якоби назовем любую матрицу вида (10) с положительными элементами, связанными условием (8). Обозначим через J множество всех таких матриц. В лемме 2 мы построили некоторое отображение

$$
\stackrel{\circ}{S}_{+} \rightarrow \mathbf{J}
$$

Очевидно, имеет место аналог теоремы Шохата-Фавара, утверждающий, что это отображение взаимно однозначно. 
3. По-прежнему считаем фиксированньми некоторую последовательность $s \in \stackrel{\circ}{S}_{+}$и порожденный ею функционал $\mathfrak{S}$. Представим $\mathfrak{S}$ в виде суммы $\mathfrak{S}^{(0)}+\mathfrak{S}^{(1)}$, где функционалы $\mathfrak{S}^{(0)}$ и $\mathfrak{S}^{(1)}$ соответствуют последовательностям

$$
\left(s_{0}, 0,0, s_{3}, 0,0, s_{6}, \ldots\right) \text { и }\left(0, s_{1}, 0,0, s_{4}, 0,0, \ldots\right) .
$$

Наряду с многочленами $H_{n}(\lambda)$ будем изучать многочлены второго рода

$$
K_{n}^{(j)}(\lambda)=\mathfrak{S}_{x}^{(j)}\left\{\frac{H_{n}(\lambda)-H_{n}(x)}{\lambda-x}\right\}, \quad n \in \mathbb{Z}_{+}, \quad j=0,1 .
$$

Рассмотрим также формальные степенные ряды

$$
f^{(j)}(\lambda)=\mathfrak{S}_{x}^{(j)}\left\{\frac{1}{\lambda-x}\right\}=\sum_{n=0}^{\infty} \frac{s_{3 n+j}}{\lambda^{3 n+j+1}}, \quad j=0,1 .
$$

Лемма 3. Степенной ряд $J_{n}^{(j)}(\lambda)=H_{n}(\lambda) f^{(j)}(\lambda)-K_{n}^{(j)}(\lambda)$ имеет вид

$$
J_{n}^{(j)}(\lambda)=\frac{\nu_{n}^{(j)}}{\lambda^{[n / 2]+1}}+\cdots,
$$

где $\nu_{n}^{(0)} \neq 0$ для четных $n, u \nu_{n}^{(1)} \neq 0$ для нечетных $n$.

ДокАЗАТЕЛЬСтво. Рассмотрим степенной ряд

$$
J_{n}(\lambda)=J_{n}^{(0)}(\lambda)+J_{n}^{(1)}(\lambda) .
$$

Тогда

$$
J_{n}(\lambda)=H_{n}(\lambda) \mathfrak{S}_{x}\left\{\frac{1}{\lambda-x}\right\}-\mathfrak{S}_{x}\left\{\frac{H_{n}(\lambda)-H_{n}(x)}{\lambda-x}\right\}=\mathfrak{S}_{x}\left\{\frac{H_{n}(x)}{\lambda-x}\right\} .
$$

Следовательно,

$$
J_{n}(\lambda)=\sum_{k=0}^{\infty} \frac{\nu_{k}}{\lambda^{k+1}}
$$

где

$$
\nu_{k}=\mathfrak{S}\left\{H_{n}(x) x^{k}\right\}= \begin{cases}0, & \text { если } k<[n / 2], \\ h_{n} m_{n}, & \text { если } k=[n / 2] .\end{cases}
$$

Остается воспользоваться инвариантностью рядов $J_{n}^{(j)}$ относительно грушшы $\mathbb{Z}_{3}$.

Доказанная лемма означает, что рациональные функции

$$
\pi_{n}^{(j)}=\frac{K_{n}^{(j)}}{H_{n}}
$$

суть совместные аппроксимации Эрмита-Паде [6] рядов (12). В частности, имеет место сходимость $\pi_{n}^{(j)} \rightarrow f^{(j)}$ при $n \rightarrow \infty, j=0,1$, по неархимедовой норме поля формальных степенных рядов.

Очевидно, что многочлены второго рода удовлетворяют тому же рекуррентному соотношению, что и многочлены $H_{n}(\lambda)$, а именно:

$$
a_{n} K_{n+1}^{(j)}(\lambda)+b_{n-2} K_{n-2}^{(j)}(\lambda)=\lambda K_{n}^{(j)}(\lambda), \quad n \in \mathbb{Z}_{+},
$$

но с другими начальными условиями:

$$
K_{-2}^{(0)}=1, \quad K_{-1}^{(0)}=0, \quad K_{0}^{(0)}=0, \quad K_{-2}^{(1)}=0, \quad K_{-1}^{(1)}=1, \quad K_{0}^{(1)}=0,
$$

если положить $b_{-2}=-1, b_{-1}=-\sqrt{a_{0}}$. 
4. Установим связь совместных аппроксимаций Эрмита-Паде (13) с двумерными непрерьвньми дробями. Исследование проведем по схеме, предложенной в [7].

Обозначим через $M$ линейное пространство размерности 2 над полем комплексных чисел, а именно: $M=\left\{\left(z^{(1)}, z^{(0)}\right): z^{(j)} \in \mathbb{C}\right\}$. Напомним, что операцию деления вектора на вектор мы определили формулой (3). Будем изучать бесконечную непрерывную дробь

$$
\frac{g_{1}}{d_{1}+\frac{g_{2}}{d_{2}+\cdot}}
$$

где $g_{n}, d_{n} \in M$ (или более общо, это векторы формальных алгебраических переменных). Обозначим через

$$
\Pi_{n}=\frac{g_{1}}{d_{1}+\ddots+\frac{g_{n}}{d_{n}}}
$$

$n$-ю подходящую дробь. Тогда

$$
\Pi_{n}=\left(\frac{\varkappa_{n}^{(1)}}{\eta_{n}}, \frac{\varkappa_{n}^{(0)}}{\eta_{n}}\right)
$$

где $\eta_{n}, \varkappa_{n}^{(1)}, \varkappa_{n}^{(0)}$ - многочлены от переменных $g_{k}^{(j)}$ и $d_{k}^{(j)}, k=1, \ldots, n, j=0,1$. Обозначим через $w_{n}(z)$ следующее дробно-линейное преобразование пространства $M$ :

$$
w_{n}(z)=\frac{g_{n}}{d_{n}+z}=\left(\frac{g_{n}^{(1)}\left(d_{n}^{(0)}+z^{(0)}\right)}{d_{n}^{(1)}+z^{(1)}}, \frac{g_{n}^{(0)}}{d_{n}^{(1)}+z^{(1)}}\right) .
$$

Тогда подходящую дробь (16) можно рассматривать как композицию дробно-линейньх преобразований

$$
\Pi_{n}=w_{1} \circ \cdots \circ w_{n}(0) .
$$

Удобно вложить линейное пространство $M$ в проективное пространство

$$
\mathbb{G}=M^{*} \backslash\{0\} / \sim
$$

где $M^{*}=\left\{\left(z^{(1)}, z^{(0)}, z^{(*)}\right): z^{(j)} \in \mathbb{C}\right\}$ - трехмерное линейное пространство над $\mathbb{C}$, и факторизация в (18) производится по отношению пропорциональности векторов. Обозначим через $\pi: M^{*} \rightarrow \mathbb{G}$ каноническую проекцию

$$
\pi:\left(z^{(1)}, z^{(0)}, z^{(*)}\right) \mapsto\left(\frac{z^{(1)}}{z^{(*)}}, \frac{z^{(0)}}{z^{(*)}}\right) .
$$

Тогда каждому дробно-линейному преобразованию $w$ проективного пространства $\mathbb{G}$ соответствует линейное преобразование $W$ линейного пространства $M^{*}$ такое, что следующая диаграмма:

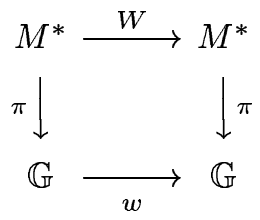


коммутативна. В частности, преобразованию $w_{n}$ соответствует линейное преобразование с матрицей

$$
W_{n}=\left(\begin{array}{ccc}
0 & g_{n}^{(1)} & g_{n}^{(1)} d_{n}^{(0)} \\
0 & 0 & g_{n}^{(0)} \\
1 & 0 & d_{n}^{(1)}
\end{array}\right)
$$

а композиции $\sigma_{n}=w_{1} \circ \cdots \circ w_{n}$ соответствует произведение матриц $\Sigma_{n}=W_{1} \cdots W_{n}$.

ЛЕмма 4. Если положить $g_{0}^{(1)}=1$, то числители и знаменатели подходящих дробей $\Pi_{n}$ будут решениями рекуррентного соотношения

$$
x_{n+1}=d_{n+1}^{(1)} x_{n}+d_{n+1}^{(0)} g_{n+1}^{(1)} x_{n-1}+g_{n+1}^{(0)} g_{n}^{(1)} x_{n-2}, \quad n \in \mathbb{Z}_{+},
$$

с начальными условиями

$$
\begin{array}{lll}
\varkappa_{-2}^{(0)}=1, & \varkappa_{-2}^{(1)}=0, & \eta_{-2}=0, \\
\varkappa_{-1}^{(0)}=0, & \varkappa_{-1}^{(1)}=1, & \eta_{-1}=0, \\
\varkappa_{0}^{(0)}=0, & \varkappa_{0}^{(1)}=0, & \eta_{0}=1 .
\end{array}
$$

ДокАЗАТЕЛЬСТво. Выбор начальных условий (20) проверяется прямьм вычислением. Далее имеем

$$
\Sigma_{n+1}=\Sigma_{n} W_{n+1}
$$

Обозначим через $y_{n}^{(1)}, y_{n}^{(2)}, y_{n}^{(3)}$ столбцы матрицы $\Sigma_{n}$. Тогда (21) равносильно соотношениям

$$
y_{n+1}^{(1)}=y_{n}^{(3)}, \quad y_{n+1}^{(2)}=g_{n+1}^{(1)} y_{n}^{(1)}, \quad y_{n+1}^{(3)}=d_{n+1}^{(0)} g_{n+1}^{(1)} y_{n}^{(1)}+g_{n+1}^{(0)} y_{n}^{(2)}+d_{n+1}^{(1)} y_{n}^{(3)}
$$

В силу $(17) x_{n}=\left(\varkappa_{n}^{(1)}, \varkappa_{n}^{(0)}, \eta_{n}\right)^{T}$ - третий столбец матрицы $\Sigma_{n}$, т.е. $x_{n}=y_{n}^{(3)}$. Исключая из системы (22) первый и второй столбцы, приходим к (19).

Как следствие получим следующее разложение в непрерывную дробь:

$$
\left(f^{(1)}(\lambda), f^{(0)}(\lambda)\right)=\frac{\left(1,-b_{-2} / a_{0}\right)}{\left(\lambda / a_{0}, 0\right)+\frac{\left(1,-b_{-1} / a_{1}\right)}{\left(\lambda / a_{1}, 0\right)+\frac{\left(1,-b_{0} / a_{2}\right)}{\left(\lambda / a_{2}, 0\right)+\ddots}} .}
$$

Действительно, для дроби (23)

$$
g_{n+1}^{(1)}=1, \quad g_{n+1}^{(0)}=-\frac{b_{n-2}}{a_{n}}, \quad d_{n+1}^{(1)}=\frac{\lambda}{a_{n}}, \quad d_{n+1}^{(0)}=0, \quad n \in \mathbb{Z}_{+} .
$$

Следовательно, соотношение (19) примет вид

$$
x_{n+1}=\frac{\lambda}{a_{n}} x_{n}-\frac{b_{n-2}}{a_{n}} x_{n-2}, \quad n \in \mathbb{Z}_{+},
$$


что совпадает с (7) и (14). Начальные условия (20) также совпадают с начальньпи условиями для многочленов $H_{n}$ и многочленов второго рода (15). Таким образом, $n$-й подходящей дробью для дроби (23) будут аппроксимации $\left(\pi_{n}^{(1)}, \pi_{n}^{(0)}\right)$. По лемме 3 они сходятся к $\left(f^{(1)}(\lambda), f^{(0)}(\lambda)\right)$.

Переходя от дроби (23) к эквивалентной дроби и обозначая $c_{n}=a_{n}^{3 / 2}$, получим разложение

$$
\left(f^{(1)}(\lambda), f^{(0)}(\lambda)\right)=\frac{(1,1)}{(\lambda, 0)+\frac{\left(c_{0}, c_{0}\right)}{(\lambda, 0)+\frac{\left(c_{1},-c_{1}\right)}{(\lambda, 0)+\frac{\left(c_{2},-c_{2}\right)}{(\lambda, 0)+\ddots}}} .}
$$

Дробь (24) можно рассматривать как двумерньй аналог непрерьвной дроби Чебышева. Она представляет собой конструктивный алгоритм вычисления отображения, обратного к отображению (11). Вычисление самого отображения (11) также сводится к разложению пары степенных рядов $\left(f^{(1)}(\lambda), f^{(0)}(\lambda)\right)$ в непрерывную дробь $(24)$ с помощью алгоритма Эйлера-Якоби-Перрона [7] (многомерного аналога алгоритма Евклида).

5. Рассмотрим обобщенную матрицу Якоби (10), в которой

$$
a_{n}=c_{n}^{2 / 3}, \quad b_{n}=\left(c_{n} c_{n+1}\right)^{1 / 3}
$$

Построим также бесконечную матрицу $B$, все элементы которой равны 0 , кроме элементов, стоящих на трех диагоналях:

$$
(B)_{n, n+3}=\alpha_{n}, \quad(B)_{n+3, n}=\beta_{n}, \quad(B)_{n+6, n}=\gamma_{n},
$$

где $n \in \mathbb{Z}_{+}$и $(B)_{n, m}$ есть элемент матрищы $B$, стоящий в $n$-й строке и в $m$-м столбце. При этом полагаем

$$
\begin{gathered}
\alpha_{n}=-\frac{2}{3}\left(c_{n} c_{n+1} c_{n+2}\right)^{2 / 3}, \quad \beta_{n}=\frac{1}{3}\left(c_{n} c_{n+1} c_{n+2}\right)^{1 / 3}\left(c_{n-1}+c_{n+1}+c_{n+3}\right) \\
\gamma_{n}=\frac{1}{3}\left(c_{n} c_{n+1} c_{n+2} c_{n+3} c_{n+4} c_{n+5}\right)^{1 / 3}
\end{gathered}
$$

где $n \in \mathbb{Z}_{+}$. Обозначим через $[A, B]=A B-B A$ коммутатор двух матриц. Прямым вычислением проверяется

Лемма 5. Последовательность положительных функиий $c_{n}=c_{n}(t)$ удовлетворяет системе дифференциальных уравнений (4) с граничными условиями (5) тогда и только тогда, когда соответствующие матриць $A$ и $B$ образуют пару Лакса, т.е. удовлетворяют уравнению $\dot{A}=[A, B]$.

Пусть последовательность $c_{n}$ ограничена, тогда $A$ и $B$ - матрицы ограниченных операторов в гильбертовом пространстве $\ell_{2}\left(\mathbb{Z}_{+}\right)$, записанные в стандартном базисе $e_{0}, e_{1}$, $e_{2}, \ldots$ В работах [8], [9] изучались спектральные свойства несимметричных операторов, заданных обобщенными матрицами Якоби. В частности, было показано как вычисляется резольвента $R_{\lambda}=(\lambda-A)^{-1}$ в терминах аппроксимаций Эрмита-Паде. В 
дальнейшем нам понадобится знать лишь нулевой и первый столбцы, а также нулевую строку матрицы $R_{\lambda}$. Для столбцов имеем формулы

$$
R_{\lambda} e_{0}=J^{(0)}, \quad R_{\lambda} e_{1}=\frac{1}{\sqrt{a_{0}}} J^{(1)}
$$

где $J^{(j)}$ - столбцы из функций второго рода $J_{n}^{(j)}$ (см. лемму 3 ). Для того чтобы найти нулевую строку матрицы $R_{\lambda}$, необходимо рассмотреть задачу, двойственную по отношению к задаче о совместных аппроксимациях Эрмита-Паде. А именно, будем изучать рекуррентное соотношение $A^{*} x=\lambda x$, где $A^{*}$ - сопряженньй оператор, или в явном виде

$$
\lambda x_{0}=b_{0} x_{2}, \quad \lambda x_{1}=a_{0} x_{0}+b_{1} x_{3}, \quad \lambda x_{2}=a_{1} x_{1}+b_{2} x_{4}, \ldots .
$$

Полагая $x_{n}=F_{n}^{(j)}(\lambda)$ и выбирая начальные условия

$$
F_{0}^{(0)}=1, \quad F_{1}^{(0)}=0, \quad F_{0}^{(1)}=0, \quad F_{1}^{(1)}=\frac{1}{\sqrt{a_{0}}}
$$

получим две последовательности многочленов $F_{n}^{(0)}(\lambda)$ и $F_{n}^{(1)}(\lambda)$ соответственно. Заметим, что последовательность многочленов $\left\{H_{n}\right\}$ и последовательность пар многочленов $\left\{\left(F_{n}^{(0)}, F_{n}^{(1)}\right)\right\}$ образуют биортонормированную систему относительно пары функционалов $\left(\mathfrak{S}^{(0)}, \mathfrak{S}^{(1)}\right)$, а именно:

$$
\mathfrak{S}^{(0)}\left\{H_{n}(\lambda) F_{m}^{(0)}(\lambda)\right\}+\mathfrak{S}^{(1)}\left\{H_{n}(\lambda) F_{m}^{(1)}(\lambda)\right\}=\left\{\begin{array}{l}
0, \text { если } n \neq m, \\
1, \text { если } n=m .
\end{array}\right.
$$

Определим многочлены второго рода формулой

$$
L_{n}(\lambda)=\mathfrak{S}_{x}^{(0)}\left\{\frac{F_{n}^{(0)}(\lambda)-F_{n}^{(0)}(x)}{\lambda-x}\right\}+\mathfrak{S}_{x}^{(1)}\left\{\frac{F_{n}^{(1)}(\lambda)-F_{n}^{(1)}(x)}{\lambda-x}\right\}
$$

и рассмотрим аппроксимации

$$
G_{n}(\lambda)=F_{n}^{(0)}(\lambda) f^{(0)}(\lambda)+F_{n}^{(1)}(\lambda) f^{(1)}(\lambda)-L_{n}(\lambda)
$$

Тогда нулевой строкой матрицы $R_{\lambda}$ будет $e_{0} R_{\lambda}=G$ - строка из аппроксимаций $G_{n}$.

Выясним как преобразуется система уравнений (4), (5) при отображении (11).

Лемма 6. Оораниченная последовательность положсительных функиий $c_{n}=$ $c_{n}(t)$ удовлетворяет системе дифференциальных уравнений (4) с граничными условиями (5) тогда и только тогда, когда соответствующая ей последовательность моментов $s_{n}=s_{n}(t)$ удовлетворяет системе дифференииальных уравнений

$$
\dot{s}_{n}=s_{n+3}-s_{n} s_{3}, \quad n \in \mathbb{Z}_{+}
$$


ДокАЗАТЕЛЬСтво. Перепишем (25) в виде

$$
\begin{aligned}
\dot{s}_{3 n} & =s_{3 n+3}-s_{3 n} s_{3}, & & n \in \mathbb{Z}_{+}, \\
\dot{s}_{3 n+1} & =s_{3 n+4}-s_{3 n+1} s_{3}, & & n \in \mathbb{Z}_{+} .
\end{aligned}
$$

Уравнения (26) равносильны следующему уравнению для производящей функции:

$$
\dot{f}^{(0)}(\lambda)=\left(\lambda^{3}-c_{0} c_{1}\right) f^{(0)}(\lambda)-\lambda^{2}
$$

а уравнения (27) равносильны уравнению

$$
\dot{f}^{(1)}(\lambda)=\left(\lambda^{3}-c_{0} c_{1}\right) f^{(1)}(\lambda)-c_{0} \lambda .
$$

Зависимость всех величин от параметра $t$ явно не указывается, но подразумевается. Из леммы 5 по индукции получим

$$
\frac{d}{d t} A^{n}=\left[A^{n}, B\right]
$$

и, следовательно, $\dot{R}_{\lambda}=\left[R_{\lambda}, B\right]$. Поскольку $f^{(0)}(\lambda)=\left(R_{\lambda}\right)_{0,0}$, то

$$
\dot{f}^{(0)}=\beta_{0} G_{3}+\gamma_{0} G_{6}-\alpha_{0} J_{3}^{(0)} .
$$

Проведем вычисления аппроксимаций:

$$
\begin{gathered}
G_{3}(\lambda)=\left(c_{0} c_{1} c_{2}\right)^{-1 / 3}\left(-c_{0} f^{(0)}(\lambda)+\lambda f^{(1)}(\lambda)\right), \\
G_{6}(\lambda)=\left(c_{0} c_{1} c_{2} c_{3} c_{4} c_{5}\right)^{-1 / 3}\left(\left(\lambda^{3}+c_{0} c_{3}\right) f^{(0)}(\lambda)-\left(c_{1}+c_{3}\right) \lambda f^{(1)}(\lambda)-\lambda^{2}\right), \\
J_{3}^{(0)}(\lambda)=\left(c_{0} c_{1} c_{2}\right)^{-2 / 3}\left(\left(\lambda^{3}-c_{0} c_{1}\right) f^{(0)}(\lambda)-\lambda^{2}\right) .
\end{gathered}
$$

Подставляя (31) в (30) и приводя подобные члены, получим (28). Аналогично доказывается равенство (29).

6. Докажем, что последовательность многочленов $H_{n}(\lambda)$ образует систему Штурма.

ЛЕмма 7. Многочлен $H_{3 n+j}(\lambda)$ имеет ровно $n$ простых нулей на интервале $(0,+\infty)$ и нуль порядка $j$ в точке $\lambda=0$, где $n \in \mathbb{Z}_{+}, j=0,1,2$. Положсительные нули многочленов $H_{n}(\lambda)$ и $H_{n+1}(\lambda)$ перемежсаются, т.е. мехду двумя последовательными нулями одного из этих многочленов лежст ровно один нуль другого многочлена.

ДокАЗАТЕЛьство. Указанное поведение многочленов в точке $\lambda=0$ непосредственно вытекает из рекуррентного соотношения (7).

Рассмотрим многочлены

$$
\Delta_{n}(\lambda)=\left|\begin{array}{cc}
H_{n}(\lambda) & K_{n}^{(0)}(\lambda) \\
H_{n+1}(\lambda) & K_{n+1}^{(0)}(\lambda)
\end{array}\right|, \quad \Delta_{n}^{*}(\lambda)=\left|\begin{array}{cc}
H_{n}(\lambda) & K_{n}^{(0)}(\lambda) \\
H_{n+2}(\lambda) & K_{n+2}^{(0)}(\lambda)
\end{array}\right|
$$

Используя рекуррентные формулы (7), (14), находим, что

$$
\begin{aligned}
a_{n} \Delta_{n}(\lambda) & =b_{n-2} \Delta_{n-2}^{*}(\lambda), & & n \geqslant 2, \\
a_{n+1} \Delta_{n}^{*}(\lambda) & =\lambda \Delta_{n}(\lambda)+b_{n-1} \Delta_{n-1}(\lambda), & & n \geqslant 1 .
\end{aligned}
$$


Из (32), (33) и начальных условий

$$
\Delta_{0}=\frac{1}{a_{0}}, \quad \Delta_{1}=0, \quad \Delta_{0}^{*}=\frac{\lambda}{a_{0} a_{1}}
$$

вытекает, что $\Delta_{n}(\lambda)$ - ненулевой многочлен с неотрицательными коэффициентами при $n \geqslant 2$. Следовательно, $\Delta_{n}(\lambda)>0$ при $\lambda>0$.

Для доказательства леммы применим индукцию. База индукции очевидна. Проведем шаг индукции. Предположим, что многочлен $H_{3 n-1}$ имеет $n-1$ простых положительных нулей $\lambda_{3 n-1, j}$, а многочлен $H_{3 n}$ имеет $n$ простых положительных нулей $\lambda_{3 n, j}$, причем эти нули перемежаются, т.е.

$$
0<\lambda_{3 n, 1}<\lambda_{3 n-1,1}<\lambda_{3 n, 2}<\cdots<\lambda_{3 n-1, n-1}<\lambda_{3 n, n} .
$$

Поскольку

$$
\Delta_{3 n-1}\left(\lambda_{3 n, j}\right)=H_{3 n-1}\left(\lambda_{3 n, j}\right) K_{3 n}^{(0)}\left(\lambda_{3 n, j}\right)>0
$$

и

$$
\Delta_{3 n}\left(\lambda_{3 n, j}\right)=-H_{3 n+1}\left(\lambda_{3 n, j}\right) K_{3 n}^{(0)}\left(\lambda_{3 n, j}\right)>0,
$$

то числа $H_{3 n-1}\left(\lambda_{3 n, j}\right)$ и $H_{3 n+1}\left(\lambda_{3 n, j}\right)$ имеют разные знаки. В силу (34)

$$
H_{3 n+1}\left(\lambda_{3 n, n}\right)<0, \quad H_{3 n+1}\left(\lambda_{3 n, n-1}\right)>0, \ldots,
$$

знаки чередуются. Следовательно, многочлен $H_{3 n+1}(\lambda)$ имеет $n$ положительных нулей вида

$$
\lambda_{3 n, 1}<\lambda_{3 n+1,1}<\lambda_{3 n, 2}<\cdots<\lambda_{3 n, n}<\lambda_{3 n+1, n} .
$$

Но в силу инвариантности он не может иметь более чем $n$ положительных нулей. Аналогичным образом происходит переход к многочлену $H_{3 n+2}(\lambda)$, а затем - к многочлену $H_{3 n+3}(\lambda)$. В последнем случае необходимо также определить знак $\operatorname{sgn} H_{3 n+3}(0)=$ $(-1)^{n+1}$, что сразу следует из рекуррентной формулы (7).

Рациональные функции

$$
\lambda^{m} \pi_{n}^{(m)}(\lambda)=\frac{\lambda^{m} K_{n}^{(m)}(\lambda)}{H_{n}(\lambda)}, \quad m=0,1
$$

в силу леммы 7 имеют следуюшие разложения в сумму простейших дробей:

$$
\lambda^{m} \pi_{3 n+k}^{(m)}(\lambda)=\mu_{3 n+k, 0}^{(m)} \cdot \frac{1}{\lambda}+\sum_{j=1}^{n} \mu_{3 n+k, j}^{(m)} \cdot \frac{3 \lambda^{2}}{\lambda^{3}-\lambda_{3 n+k, j}^{3}} .
$$

Коэффициенты $\mu_{n, j}^{(m)}$ разложения (35) будем называть коэффициентами Кристоффеля.

Лемма 8. Все коэффичиенты Кристоффеля строго полоэсительнь, за исключением $\mu_{3 n, 0}^{(0)}=0, \mu_{3 n, 0}^{(1)}=0, \mu_{3 n+1,0}^{(1)}=0$. 
ДоказАТЕЛЬСтво. Рассмотрим лиш коэффициенты $\mu_{3 n, j}^{(0)}$, где $j=1, \ldots, n$. Для остальных коэффициентов доказательство аналогично. Согласно лемме 7

$$
H_{3 n}^{\prime}\left(\lambda_{3 n, n}\right)>0, \quad H_{3 n}^{\prime}\left(\lambda_{3 n, n-1}\right)<0, \ldots,
$$

знаки чередуются. Из доказательства леммы 7 следует, что

$$
K_{3 n}^{(0)}\left(\lambda_{3 n, n}\right)>0, \quad K_{3 n}^{(0)}\left(\lambda_{3 n, n-1}\right)<0, \ldots,
$$

знаки чередуются, причем чередование знаков то же, что в (36). Вычисляя коэффициенты Кристоффеля как вычеты в простых полюсах, находим

$$
\mu_{3 n, j}^{(0)}=\underset{\substack{\text { res } \\ \lambda=\lambda_{3 n, j}}}{(0)} \pi_{3 n}^{(\lambda)}=\frac{K_{3 n}^{(0)}\left(\lambda_{3 n, j}\right)}{H_{3 n}^{\prime}\left(\lambda_{3 n, j}\right)}>0
$$

7. Обозначим через $\mathfrak{M}$ множество всех конечных положительных борелевских мер на $\Delta$, имеющих конечные степенные моменты любого порядка. Построим дискретные меры $d \mu_{3 n+k}^{(m)} \in \mathfrak{M}$, сосредоточив в каждой из точек $\lambda_{3 n+k, j} \varepsilon^{l}, l=0,1,2$, массу $\mu_{3 n+k, j}^{(m)}$, где $j=1, \ldots, n$, и массу $\mu_{3 n+k, 0}^{(m)}$ в точке $\lambda=0$. По построению эти меры инвариантны относительно группы $\mathbb{Z}_{3}$. Разложение (35) можно переписать в виде

$$
\lambda^{m} \pi_{n}^{(m)}(\lambda)=\int_{\Delta} \frac{d \mu_{n}^{(m)}(x)}{\lambda-x} .
$$

В силу леммы 3 меры $d \mu_{n}^{(m)}$ решают следующую усеченную проблему моментов:

$$
\int_{\Delta} x^{3 k} d \mu_{n}^{(m)}(x)=s_{3 k+m}, \quad k=0, \ldots, n+\left[\frac{n}{2}\right]-1, \quad m=0,1 .
$$

В частности, все меры $d \mu_{n}^{(0)}, n \geqslant 1$, вероятностные, а все меры $d \mu_{n}^{(1)}, n \geqslant 2$, имеют полную вариацию $s_{1}$. По теореме Хелли из последовательностей мер $\left\{d \mu_{n}^{(m)}\right\}_{n=0}^{\infty}$ можно выделить подпоследовательности, сходящиеся в $*$-слабой топологии к некоторым мерам $d \mu^{(m)} \in \mathfrak{M}$, которые решают следующую проблему моментов:

$$
\int_{\Delta} x^{3 k} d \mu^{(m)}(x)=s_{3 k+m}, \quad k \in \mathbb{Z}_{+}, \quad m=0,1 .
$$

Тем самым, степенные ряды

$$
\lambda^{m} f^{(m)}(\lambda), \quad m=0,1,
$$

будут асимптотическими разложениями функций

$$
\int_{\Delta} \frac{d \mu^{(m)}(x)}{\lambda-x}, \quad m=0,1
$$

голоморфных на открытом множестве $\mathbb{C} \backslash \Delta$.

В случае неограниченного оператора $A$ встает задача об описании всех решений проблемы моментов (37) в терминах расширений этого оператора. В настоящей работе мы 
рассматриваем лиш случай ограниченных операторов. В этом случае из определенности проблемы моментов Хаусдорфа [10] вытекает единственность решения задачи (37), причем последовательности мер $d \mu_{n}^{(m)}$ слабо сходятся к этому решению. Обозначим через $\rho$ наименьший радиус круга $|\lambda| \leqslant \rho$, содержащего общий носитель мер $d \mu^{(m)}$, и через $\Delta_{\rho}$ - пересечение этого круга с $\Delta$. Тогда степенные ряды (38) будут рядами Лорана функций $(39)$, голоморфных в области $\overline{\mathbb{C}} \backslash \Delta_{\rho}$, сходящимися при $|\lambda|>\rho$. Из слабой сходимости мер вытекает аналог теоремы Маркова, а именно: при $n \rightarrow \infty$ совместные апроксимации Эрмита-Паде $\lambda^{m} \pi_{n}^{(m)}(\lambda)$ сходятся к функциям (39) равномерно внутри области $\overline{\mathbb{C}} \backslash \Delta_{\rho}$. Меры $d \mu^{(m)}$ естественно назьвать спектральными мерами оператоpa $A$.

Следует подчеркнуть, что в отличии от случая самосопряженных операторов носитель спектральных мер не совпадает со спектром оператора. В [9] был рассмотрен пример оператора $A$ с элементами $a_{n}=1, b_{n}=1$, носителем спектральных мер которого является множество $\Delta_{\rho}$, где $\rho=3 \cdot 2^{-2 / 3}$, а спектром служит часть комплексной плоскости, ограниченная трехлепестковой розой $r=2 \cos 3 \varphi$.

Другая интересная на наш взгляд задача состоит в описании множества $U$ всех производных Радона-Никодима $u=d \mu^{(1)} / d \mu^{(0)}$. Здесь мы можем привести лиш одно простое достаточное условие принадлежности функции $u$ классу $U$, которое легко доказывается методами работы [11]. А именно, если

i) $(1, u)$ и $\left(u, x^{3}\right)$ являются АТ-системами на промежутке $[0,+\infty)$;

ii) для некоторого $0<\alpha<3$ имеем

$$
0<\varliminf_{x \rightarrow+\infty} u(x) x^{-\alpha} \leqslant \varlimsup_{x \rightarrow+\infty} u(x) x^{-\alpha}<+\infty,
$$

то для любой меры $d \mu \in \mathfrak{M}$, имеющей бесконечньй носитель, последовательность моментов

$$
s_{3 n}=\int_{\Delta} x^{3 n} d \mu(x), \quad s_{3 n+1}=\int_{\Delta} x^{3 n} u(x) d \mu(x), \quad s_{3 n+2}=0
$$

будет позитивной, т.е. $u \in U$. Заметим, что из одного лишь условия і) следует несингулярность последовательности (40), но не следует, вообще говоря, ее позитивность. Этот факт вытекает из рассмотренного нами в работе [12] примера мер

$$
d \mu^{(0)}(x)=e^{-x} d x, \quad d \mu^{(1)}(x)=x e^{-2 x} d x .
$$

8. ДокаЗАТЕЛЬСТво тЕоремы. В силу (24) непрерьвная дробь в (6) сходится к вектору $\left(f^{(1)}, f^{(0)}\right)$, а из п. 7 вытекает, что соответствующие функции имеют интегральное представление (39). По лемме 6 система (4) равносильна системе (25), которую можно переписать в виде $\dot{s}_{n}^{*}=s_{n+3}^{*}$, где $s_{n}^{*}=s_{n} s_{0}^{*}, n \in \mathbb{Z}_{+}$. Следовательно,

$$
s_{3 n+m}^{*}=\int_{\Delta} x^{3 n} d \mu^{*(m)}(x, t), \quad n \in \mathbb{Z}_{+}, \quad m=0,1,
$$

где меры $d \mu^{*(m)}$ удовлетворяют дифференциальным уравнениям

$$
\frac{\partial}{\partial t} d \mu^{*(m)}=x^{3} d \mu^{*(m)},
$$

которые при заданных начальных условиях имеют единственное решение

$$
d \mu^{*(m)}(x, t)=e^{x^{3} t} d \mu^{*(m)}(x, 0) .
$$

Теорема доказана. 
9. Приведем пример решения цепочки (4) с неограниченными начальными данными. Пусть

$$
d \mu^{(0)}(x, t)=\frac{1}{x} d \mu^{(1)}(x, t)=d(t) e^{-x^{3} t} d x, \quad x>0,
$$

где $d(t)$ - нормировочньй множитель. Тогда многочленами $H_{n}$ будут обобщенные многочлены Эрмита, которые изучались нами в [13] в связи с физическими приложениями. Эти многочлены могут быть определены формулами Родрига

$$
e^{-\lambda^{3} t} H_{2 n+m}(\lambda)=d_{2 n+m}(t)\left(\frac{d}{d \lambda}\right)^{n}\left(\lambda^{m} e^{-\lambda^{3} t}\right), \quad n \in \mathbb{Z}_{+}, \quad m=0,1,
$$

которые легко доказьваются интегрированием по частям. При этом $d_{n}(t)$ - нормировочные постоянные. Из (41) находим

$$
c_{2 n}(t)=\frac{\Gamma(2 / 3)}{\Gamma(1 / 3)} t^{-1 / 3}, \quad c_{2 n+1}(t)=\frac{n+1}{3} \frac{\Gamma(1 / 3)}{\Gamma(2 / 3)} t^{-2 / 3} .
$$

Следовательно, $\dot{c}_{n}=-c_{n}\left(c_{n+1} c_{n+2}-c_{n-1} c_{n-2}\right)$. Таким образом, $c_{n}\left(t_{0}-t\right)-$ решение цепочки на промежутке $0 \leqslant t<t_{0}$.

\section{СПИСОК ЦИТИРОВАННОЙ ЛИТЕРАТУРЫ}

[1] Moser J. K. Three integrable Hamiltonian systems connected with isospectral deformations // Adv. Math. 1975. V. 16. P. 197-220.

[2] Богоявленский О.И. Некоторые конструкции интегрируемых динамических систем // Изв. АН СССР. Сер. матем. 1987. Т. 51. № 4. С. 737-766.

[3] Богоявленский О.И. Интегрируемые динамические системы, связанные с уравнением КдФ // Изв. АН СССР. Сер. матем. 1987. Т. 51. №6. С. 1123-1141.

[4] Осипов А. С. Дискретный аналог уравнения Кортевега-де Фриза (КдФ): интегрирование методом обратной задачи // Матем. заметки. 1994. Т. 56. №6. С. 141-144.

[5] Юрко В.А. Об интегрировании нелинейных динамических систем методом обратной спектральной задачи // Матем. заметки. 1995. Т. 57. №6. С. 945-949.

[6] Никишин Е. М., Сорокин В.Н. Рациональные аппроксимации и ортогональность. М.: Наука, 1988.

[7] Парусников В.И. Алгоритм Якоби-Перрона и совместные приближения функций // Матем. сб. 1981. Т. 114. №2. С. 322-333.

[8] Калягин В. А. Аппроксимации Эрмита-Паде и спектральная теория несимметричных операторов // Матем. сб. 1994. Т. 185. №6. С. 79-100.

[9] Sorokin V. N. Connection between matrix Hermite-Padé approximation and matrix continued fraction in the example of a particular Toeplitz matrix. Reprint № ANO-346. Lille: Universite des Sciences et Technologies de Lille, 1995.

[10] Ахиезер Н. И. Классическая проблема моментов. М.: Физматгиз, 1961.

[11] Никишин Е. М. О совместных аппроксимациях Паде // Матем. сб. 1980. Т. 113. № 4. C. $499-519$.

[12] Сорокин В. Н. Сходимость совместных аппроксимаций Паде к функциям стилтьесовского типа // Изв. вузов. Матем. 1987. № 7. С. 48-56.

[13] Вшивцев А. С., Сорокин В.Н. Теория возмущений для уравнения Шрёдингера с полиномиальным потенциалом // Изв. вузов. Физика. 1994. № 1. С. 95-101. 